\title{
Chitosan Oligosaccharides Inhibit/Disaggregate Fibrils and Attenuate Amyloid $\beta$-Mediated Neurotoxicity
}

\author{
Xueling Dai ${ }^{1, \dagger}$, Wanqi Hou ${ }^{2, \dagger}$, Yaxuan Sun ${ }^{1}$, Zhaolan Gao ${ }^{1}$, Shigong Zhu ${ }^{3}$ and \\ Zhaofeng Jiang 1 ,*
}

1 Beijing Key Laboratory of Bioactive Substances and Functional Foods, Beijing Union University, Beijing 100191, China; E-Mails: xueling@buu.edu.cn (X.D.); sunxx@buu.edu.cn (Y.S.); zhaolan@buu.edu.cn (Z.G.)

2 College of Life Sciences, Capital Normal University, Beijing 100048, China; E-Mail: bluefire828@126.com

3 Department of Physiology and Pathophysiology, Peking University School of Basic Medical Sciences, Beijing 100191, China; E-Mail: sgzhu@bjmu.edu.cn

$\dagger$ These authors contributed equally to this work.

* Author to whom correspondence should be addressed; E-Mail: zfjiang6@gmail.com; Tel.: +86-10-6200-4534; Fax: +86-10-6238-8926.

Academic Editor: Vassilios Roussis

Received: 6 March 2015 / Accepted: 3 May 2015 / Published: 8 May 2015

\begin{abstract}
Alzheimer's disease (AD) is characterized by a large number of amyloid- $\beta$ (A $\beta$ ) deposits in the brain. Therefore, inhibiting $A \beta$ aggregation or destabilizing preformed aggregates could be a promising therapeutic target for halting/slowing the progression of AD. Chitosan oligosaccharides (COS) have previously been reported to exhibit antioxidant and neuroprotective effects. Recent study shows that COS could markedly decrease oligomeric $A \beta$-induced neurotoxicity and oxidative stress in rat hippocampal neurons. However, the potential mechanism that COS reduce $A \beta$-mediated neurotoxicity remains unclear. In the present study, our findings from circular dichroism spectroscopy, transmission electron microscope and thioflavin $\mathrm{T}$ fluorescence assay suggested that $\mathrm{COS}$ act as an inhibitor of $\mathrm{A} \beta$ aggregation and this effect shows dose-dependency. Moreover, data from thioflavin $\mathrm{T}$ assay indicated that COS could significantly inhibit fibrils formation and disrupt preformed fibrils in a dose-dependent manner. Furthermore, the addition of COS attenuated A $\beta 1-42$-induced neurotoxicity in rat cortical neurons. Taken together, our results demonstrated for the first time that COS could inhibit
\end{abstract}


A $\beta 1-42$ fibrils formation and disaggregate preformed fibrils, suggesting that COS may have anti-A $\beta$ fibrillogenesis and fibril-destabilizing properties. These findings highlight the potential role of $\operatorname{COS}$ as novel therapeutic agents for the prevention and treatment of $\mathrm{AD}$.

Keywords: Alzheimer's disease; amyloid- $\beta$ peptide; chitosan oligosaccharides; aggregation; neurotoxicity

\section{Introduction}

Alzheimer's disease $(\mathrm{AD})$ is a progressive, age-related neurodegenerative disorder, characterized by the deposition of amyloid $\beta$ (A $\beta$ ) forming senile plaques (SPs), intracellular neurofibrillary tangles (NFTs) and neuronal loss in the brain of AD patients [1,2]. Although there remains debate on whether $\mathrm{A} \beta$ accumulation is a cause or an effect of $\mathrm{AD}, \mathrm{A} \beta$ overproduction and deposition appears to be the earliest identifiable event associated with the development of $\mathrm{AD}$ [3]. The fundamental pathogenic occurrence in $\mathrm{AD}$ is the misfolding and aggregation process of $\mathrm{A} \beta$ peptides, which triggers a cascade of events that result in the occurrence of amyloid plaques, neuronal degeneration and dementia [4]. To date, acetylcholinesterase inhibitors (i.e., donepezil, rivastigmine, and galantamine) and the NMDA receptor antagonist (memantine) have been approved for treating patients with $\mathrm{AD}$, though these therapies exhibit a modest benefit in improving cognitive deficits [5,6]. Currently, there is no effective therapeutic agent available to treat AD.

Given the growing prevalence and poor prognosis of AD, there is an urgent need to develop novel, effective therapeutic approaches that not only ameliorating the disease symptoms but also slowing down or inhibiting the underlying neurodegenerative process. Recently, particular attention has been paid to small chemical compounds that derived from natural products, due to the ease of accessibility and structural modification [7]. Chitosan oligosaccharides (COS), the hydrolysis products from chitosan that composed of linear polymers of $\beta$-1-4-linked D-glucosamine [8], were shown to have a wide range of biological activities including immunity regulation, anti-oxidant, anti-cancer and anti-inflammatory properties [9-12]. Our recent study suggested that COS could markedly attenuate oligomeric $A \beta 1-42$-induced neurotoxicity via repression of oxidative stress and blocking $A \beta$-mediated phosphorylation of c-Jun $N$-terminal kinase [13]. However, no existing literature states whether COS have direct effects on $A \beta$ aggregation and deposition, which is an important contributor to the occurrence of $\mathrm{AD}$. In the present study, we investigated the potential effect of COS on preventing monomeric $A \beta$ aggregation, dissembling preformed fibrils and $A \beta$-mediated neurotoxicity.

\section{Results}

\subsection{Circular Dichroism Analysis of COS Influences on AB}

Circular dichroism (CD) spectroscopy provides a general indicator for the increase in $\beta$-structure content that accompanies the aggregation of the A $\beta$ peptide [14]. To investigate the structural changes of the peptide, CD spectroscopy in the far UV region (190-240 nm) was applied to detect the changes 
in secondary structure of A $\beta 1-42$ (A $\beta 42)$ alone or peptides coincubated with COS for $48 \mathrm{~h}$ respectively. In the absence of COS, the $\mathrm{CD}$ spectra of $\mathrm{A} \beta$ exhibited a transition from unstructured random coil to $\beta$-sheet, which displayed a minimum negative band at around $216 \mathrm{~nm}$ upon 24 or $48 \mathrm{~h}$ incubation (Figure 1A). However, as shown in Figure 1B, the CD spectra of $50 \mu \mathrm{M} A \beta 42$ coincubated with 2.5 or $5.0 \mathrm{mg} / \mathrm{mL}$ COS for $48 \mathrm{~h}$ suggested a largely disordered structure. The conformational conversion of $50 \mu \mathrm{M} \mathrm{A} \beta 42$ from physiological unfolded random coil to $\beta$-sheet structure was partly suppressed by $2.5 \mathrm{mg} / \mathrm{mL}$ COS addition, manifested by decreased negative band at $\sim 216 \mathrm{~nm}$ compared to A $\beta 42$ alone; while with addition of $5.0 \mathrm{mg} / \mathrm{mL}$ COS, the spectrum appeared a broader negative band at $\sim 200 \mathrm{~nm}$ suggesting the secondary structure of $A \beta$ is mainly random coil [15]. These results suggest that $\operatorname{COS}$ could prevent $A \beta$ from converting into $\beta$-sheet structure in a dose-dependent manner, where COS at a concentration of $5.0 \mathrm{mg} / \mathrm{mL}$ was more effective than that of $2.5 \mathrm{mg} / \mathrm{mL}$ in inhibiting $\beta$-structure formation.

A

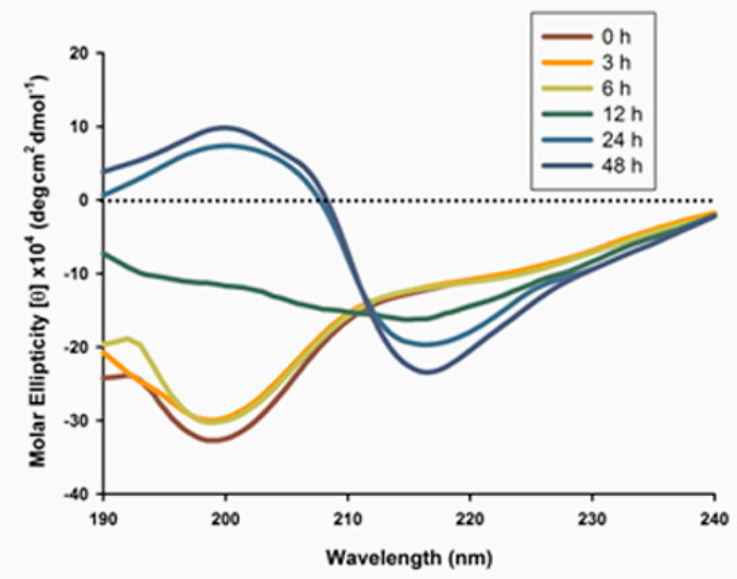

B

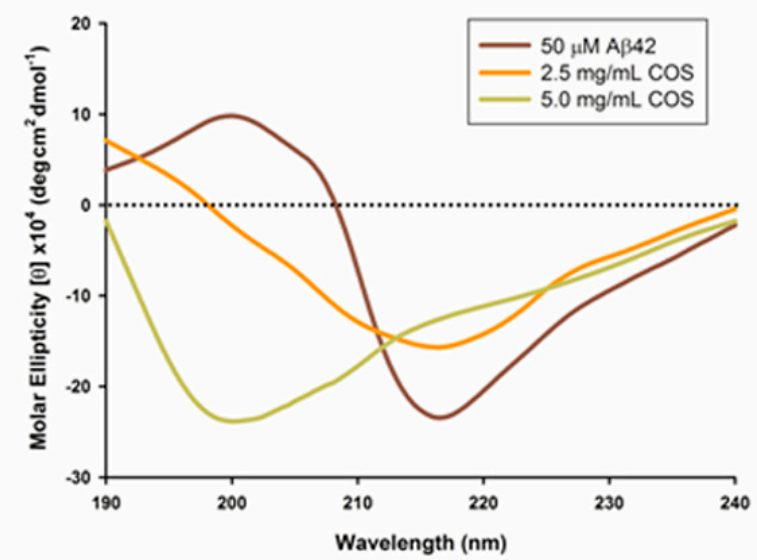

Figure 1. Circular Dichroism (CD) spectroscopy of $A \beta 42$. Amyloid- $\beta$ (A $\beta$ ) peptides were diluted with $10 \mathrm{mM}$ phosphate buffer to a final concentration of $50 \mu \mathrm{M}$ in each sample. (A) CD spectra of A $\beta 42$ upon incubation were recorded in the UV region (190-240 nm) at $0,3,6,12,24$ and $48 \mathrm{~h}$ respectively; (B) $\mathrm{CD}$ spectra of $50 \mu \mathrm{M} \mathrm{A} \beta 42$ were recorded in the UV region (190-240 nm) upon coincubation with 2.5 or $5.0 \mathrm{mg} / \mathrm{mL}$ chitosan oligosaccharides (COS) (when present) respectively for $48 \mathrm{~h}$. Data were the average of six runs and represented as rainbow color curves after being smoothed.

\subsection{Morphologies of A Aggregates Visualized by Transmission Electron Microscope}

Transmission electron microscope (TEM) was employed to investigate the morphologies of A $\beta$ in the presence or absence of COS during the time course of $0-48 \mathrm{~h}$ at $37^{\circ} \mathrm{C}$ (Figure 2). Samples from $\mathrm{CD}$ assay where $50 \mu \mathrm{M}$ monomeric $\mathrm{A} \beta 42$ solutions in the presence or absence of 2.5 or $5.0 \mathrm{mg} / \mathrm{mL}$ COS diluted by water in the ratio of 1:5 were taken for TEM. As shown in Figure 2A, TEM analysis of monomeric fractions confirmed the absence of any aggregated $A \beta$ in these preparations (monomeric $\mathrm{A} \beta$ is not detectable by TEM) [16]. After incubation at $37{ }^{\circ} \mathrm{C}$ for $12 \mathrm{~h}$ (Figure 2B), TEM image of $10 \mu \mathrm{M}$ A $\beta 42$ alone showed aggregates of various morphologies including short and branched protofibrils (10-15 $\mathrm{nm}$ in diameter and $<200 \mathrm{~nm}$ in length), curvilinear aggregates and small spherical 
structures. Extensive long and branched fibrils with an average diameter of $\sim 15 \mathrm{~nm}$ and an average length of $\sim 1.5 \mu \mathrm{m}$ (Figure $2 \mathrm{C}$ ) were observed after $24 \mathrm{~h}$ incubation, and those aggregates prolonged into more abundant mature fibrils and produced a dense network of fibrils with an average length of $\sim 5 \mu \mathrm{m}$ (48 h, Figure 2D). Thus, morphology of A $\beta 42$ alone appeared characteristic fibrillization upon incubation which is in agreement with $C D$ results. However, the amount of fibrils appeared to decrease with increasing concentration of COS when $10 \mu \mathrm{M}$ monomeric $\mathrm{A} \beta 42$ was coincubated with COS for $48 \mathrm{~h}$ (Figure 2E,F). $1.0 \mathrm{mg} / \mathrm{mL}$ COS (Figure 2E) was more effective than $0.5 \mathrm{mg} / \mathrm{mL}$ COS (Figure 2F) in inhibiting fibrils formation. These findings obtained from TEM images were consistent with data from CD spectroscopy, suggesting that COS may have a direct inhibitory effect on the fibrillization process of $\mathrm{A} \beta 42$ from its monomeric form into fibrils.
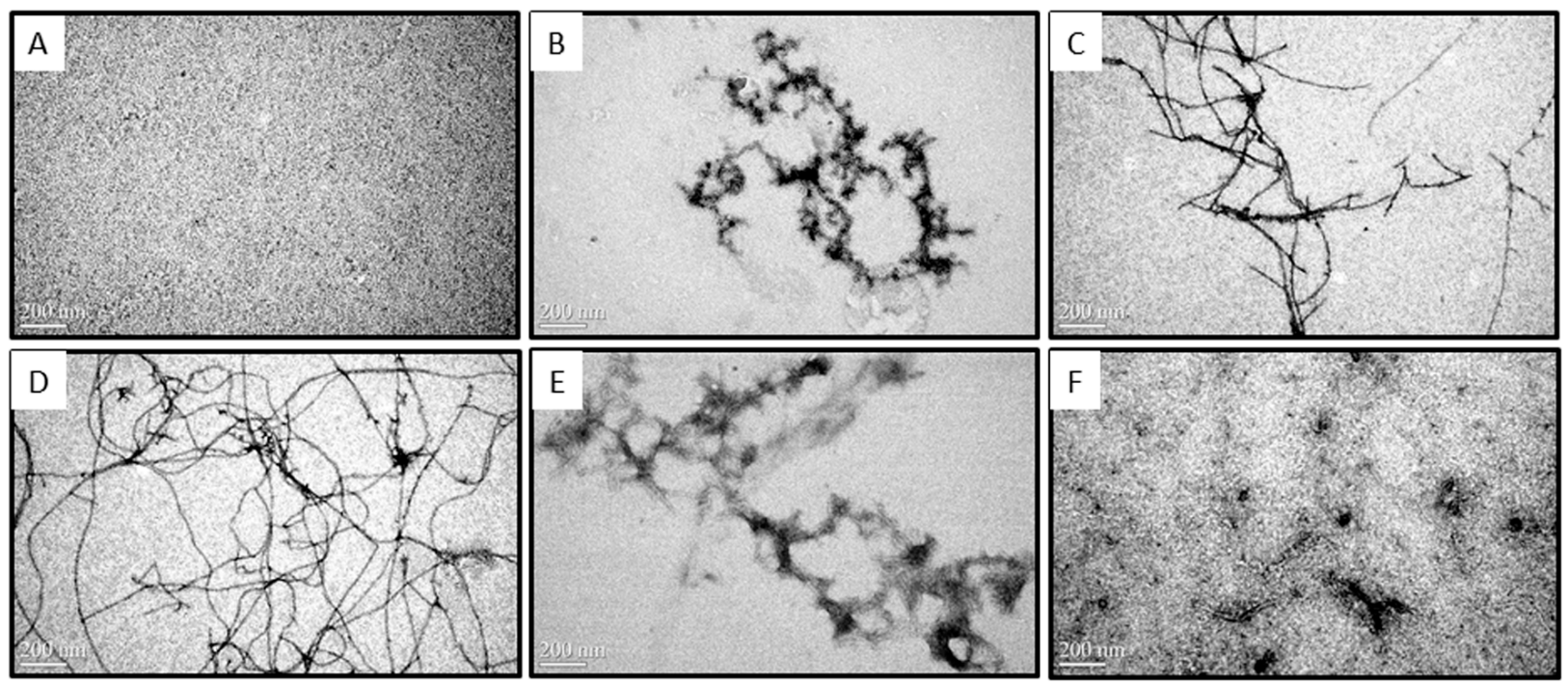

Figure 2. Effect of $\operatorname{COS}$ on $A \beta$ aggregates visualized by Transmission Electron Microscopy (TEM). Representative TEM images of monomeric A $\beta 42$ fractions soon after preparation (A) and fibrils formed by monomeric A 342 after $12 \mathrm{~h}(\mathbf{B}), 24 \mathrm{~h}(\mathbf{C})$, and $48 \mathrm{~h}$ (D) of incubation at $37{ }^{\circ} \mathrm{C}(10 \mu \mathrm{M} \mathrm{A} \beta 42$, without agitation); Representative images of monomeric Aß42 $(10 \mu \mathrm{M})$ coincubated with $0.5 \mathrm{mg} / \mathrm{mL}(\mathbf{E})$ or $1.0 \mathrm{mg} / \mathrm{mL} \operatorname{COS}(\mathbf{F})$ for $48 \mathrm{~h}$ at $37^{\circ} \mathrm{C}$ were visualized by TEM. The scale bar $(200 \mathrm{~nm})$ is shown in the lower left of the images. Magnification: $80,000 \times$.

\subsection{COS Inhibited and Disassembled A $\beta$ Fibrils in Vitro}

Thioflavin T (ThT) has been widely used as an amyloidophilic dye to detect the $\beta$-sheet structure and aggregates. To examine the inhibitory effect of COS on A $\beta$ fibrillization, samples from CD assay where $50 \mu \mathrm{M}$ monomeric $\mathrm{A} \beta 42$ solutions in the presence of 2.5 or $5.0 \mathrm{mg} / \mathrm{mL}$ COS diluted by water in the ratio of $2: 5$ were incubated at $37{ }^{\circ} \mathrm{C}$ for $48 \mathrm{~h}$. The kinetic changes of $\mathrm{A} \beta$ aggregation in the presence or absence of COS were monitored. As shown in Figure 3A, the ThT fluorescence intensity of COS alone did not change during the time course of $48 \mathrm{~h}$ incubation, suggesting that COS had no effect on disturbing the fluorescence intensity. ThT fluorescence intensity of $20 \mu \mathrm{M}$ A $\beta 42$ alone increased from 0 to $48 \mathrm{~h}$, and finally reached a high plateau after $48 \mathrm{~h}$ incubation. As expected, COS 
markedly reduced the ThT fluorescence intensity compared to $\mathrm{A} \beta 42$ alone in a dose-dependent manner, implying the inhibitory effect of $\operatorname{COS}$ on $A \beta$ fibrils formation.
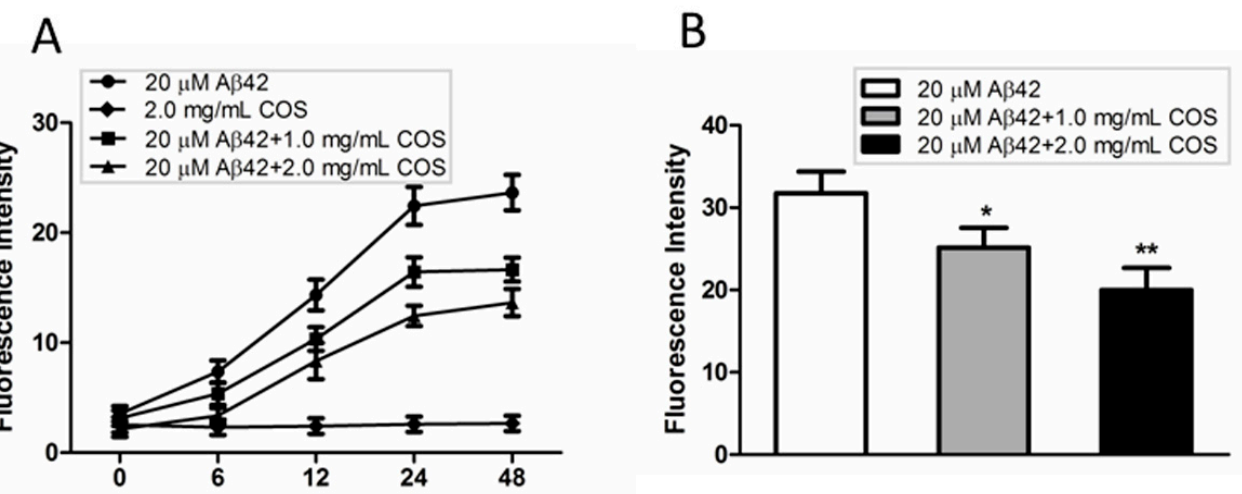

Figure 3. Effect of COS on $A \beta$ fibrils formation. (A) Time-dependent thioflavin $T$ (ThT) fluorescence intensity changes for $20 \mu \mathrm{M} A \beta 42$ incubated with different concentration of $\operatorname{COS}(1.0$ and $2.0 \mathrm{mg} / \mathrm{mL}$ respectively), as compared to $2.0 \mathrm{mg} / \mathrm{mL} \operatorname{COS}$ or $20 \mu \mathrm{M} \mathrm{A} \beta 42$ alone; (B) Disaggregative effect of COS (1.0 and $2.0 \mathrm{mg} / \mathrm{mL}$ respectively) on preformed $\mathrm{A} \beta$ fibrils. Data were represented as mean \pm SEM of three independent experiments. $* * p<0.01, * p<0.05$ vs. A $\beta 42$ alone.

We also investigated whether COS could disrupt preformed A $\beta$ fibrils using the ThT assay. As shown in Figure 3B, ThT fluorescence intensity of samples where COS were coincubated with preformed $\mathrm{A} \beta 42$ fibrils was significantly reduced in a concentration-dependent manner as compared with A $\beta 42$ fibrils alone, indicating that COS could partly disaggregate the preformed A $\beta 42$ fibrils. Taken together, these findings clearly demonstrated the effect of COS on preventing A $\beta 42$ monomers from developing into fibrillary amyloid, and also dissembling preformed fibrils.

\subsection{COS Attenuated AB42-Induced Neurotoxicity}

To determine the effect of COS on A 342 -induced toxicity, 3-[4,5-dimethylthiazol-2-yl]-2,5diphenyltetrazolium bromide (MTT) assay was conducted to compare the cell viability of A $\beta$, COS, $\mathrm{A} \beta-\mathrm{COS}$ on cortical neurons. As shown in Figure 4A, when neurons were incubated with COS alone at $0.5 \mathrm{mg} / \mathrm{mL}$, no significant decline in cell viability was observed as compared to control $(p>0.05)$, suggesting that COS are nontoxic to neurons. In contrast, the cell viability of neurons exposed to $5 \mu \mathrm{M}$ $\mathrm{A} \beta$ for $48 \mathrm{~h}$ decreased to $\sim 75.8 \%$ of the control $(p<0.01)$, while the viability of neurons treated with both $5 \mu \mathrm{M} \mathrm{A} \beta 42$ and $0.5 \mathrm{mg} / \mathrm{mL}$ COS for $48 \mathrm{~h}$ was up to $\sim 91.0 \%$. These results indicated that COS markedly protected neurons from $A \beta$-induced neurotoxicity.

A $\beta 42$-induced apoptosis was examined by Annexin V and PI double staining. As shown in Figure 4B, apoptotic cells were significantly increased following A $\beta 42$ treatment for $48 \mathrm{~h}$ compared to that in control group, suggesting that $A \beta 42$ treatment could lead to neuronal apoptosis. The addition of $0.5 \mathrm{mg} / \mathrm{mL}$ COS effectively rescued neurons from apoptosis induced by $\mathrm{A} \beta 42$ ( $p<0.01$ vs. $5 \mu \mathrm{M} \mathrm{A} \beta 42$ group), indicating that apoptosis was involving in the protective effect of $\mathrm{COS}$ on $\mathrm{A} \beta 42$-induced cytotoxicity. 

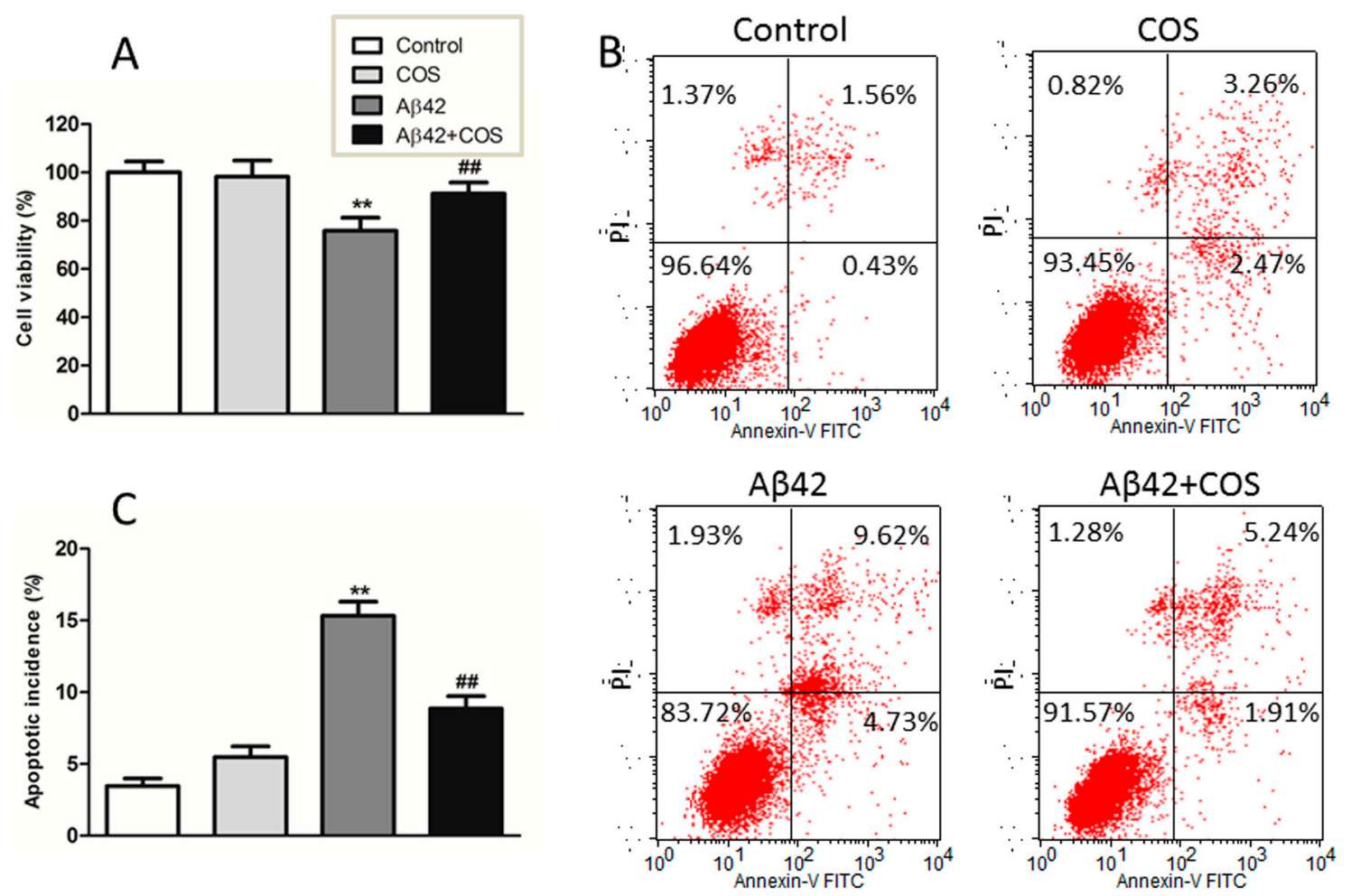

Figure 4. Effect of $\mathrm{COS}$ on $\mathrm{A} \beta 42$-induced neurotoxicity in cortical neurons. (A) Neurons were treated with $5 \mu \mathrm{M} \mathrm{A} \beta 42$ with or without addition of $0.5 \mathrm{mg} / \mathrm{mL}$ COS for $48 \mathrm{~h}$, the cell viability was determined by the MTT assay; (B) Representative graphs obtained by flow cytometry using double staining with Annexin V-FITC and PI; (C) The apoptotic incidence of rat cortical neurons exposed to $5 \mu \mathrm{M} A \beta 42$ in the presence or absence of $0.5 \mathrm{mg} / \mathrm{mL}$ COS for $48 \mathrm{~h}$. Results were expressed as the percentage of apoptotic cells that include neurons in early and late apoptotic phases. Data were expressed as means \pm SEM of three independent experiments. ${ }^{* *} p<0.01$ vs. Control, ${ }^{\#} p<0.01 v s$. A $\beta 42$ group.

\section{Discussion}

The formation of SPs in AD brain is a multiple process that involves abnormal aggregation of $\mathrm{A} \beta$ peptides from soluble unstructured monomers to various physical forms including $\beta$-sheet rich oligomers, protofibrils and insoluble fibrils [17]. Thus, any step in the process of $A \beta$ production, aggregation and clearance could be considered as a potential therapeutic target $[18,19]$. On the other hand, since neurotoxicity is mainly associated with the formation of $A \beta$ aggregates with $\beta$-sheet structure, the search for anti-aggregation and $\beta$-sheet disrupting compounds provides a potential therapeutic approach to treat $\mathrm{AD}$ [20]. Although significant efforts and progresses have been made to inhibit $A \beta$ aggregation, reduce the existed aggregation from brain or repress the initial conformational change of $A \beta$ into its pathological $\beta$-sheet formation, these strategies have not produced any effective pharmaceutical agent to date.

The formation of $A \beta$ fibrils undergoes a structural transition from random coil to organized $\beta$-sheet conformation [21]. In this study, CD spectroscopy revealed that COS inhibited the aggregation of $\mathrm{A} \beta 42$ into $\beta$-sheet structure in a dose-dependent manner. Morphological changes of $A \beta 42$ coincubated with COS detected by TEM were in parallel with CD spectroscopy. Further, ThT fluorescence assay 
confirmed that COS could effectively inhibit A $\beta 42$ fibrils formation, and disrupt the preformed fibrils. Trehalose, a simple disaccharide that has a similar structure to chitobiose, was shown to dose-dependently inhibit A $\beta 40 / 42$ aggregation and dissociate preformed aggregates partly due to the formation of $\mathrm{H}$-bonds between trehalose and $\mathrm{A} \beta$ that suppress the interpeptide hydrogen bonding [22,23]. Properties of COS that inhibit A $\beta$ aggregation and disassemble the preformed fibrils are likely a consequence of direct interaction with $A \beta$. One potential explanation for the inhibitory effect of COS can be obtained by thermodynamic reasoning. Probably, when COS were added to the monomeric peptide solutions, COS molecules altered the environment of surrounding water molecules, rendering aggregation less energetically favorable. As COS molecules would essentially isolate the water molecules surrounding the protein, thereby blocking the gain in entropy from release of this structured water layer. However, further research should be conducted to elucidate the precise mechanism by which COS interact with $A \beta$.

Either the oligomeric or fibrillar $A \beta$ shows neurotoxicity stronger than its monomeric form, and mounting evidence supports toxic $\mathrm{A} \beta$ oligomers as the drivers of neurodegeneration [24]. Therefore, inhibiting monomeric $A \beta$ aggregation into neurotoxic species or dissembling mature fibrils into non-toxic species is crucial to prevent and treat $\mathrm{AD}$ [25]. It is proposed that an efficient inhibitor should block the growth of fibrils as well as interfere with the neurotoxicity of $A \beta$ [26]. In this study, COS coincubated with monomeric $\mathrm{A} \beta 42$ for $48 \mathrm{~h}$ significantly reduced cytotoxicity in rat cortical neurons, manifested by increased cell viability and reduced apoptotic incidence. Together, these findings indicate that the preventive effect of COS is associated with its inhibitory effects on $A \beta$ misfolding by inhibiting $A \beta$ aggregation formation and probably disrupting $A \beta$ fibrils. These data extended previous work on the neuroprotective mechanism of COS against AD.

In summary, the present study along with other studies suggest the effect of COS in inhibiting aggregation and mitigating the toxicity of $\mathrm{A} \beta$, which shed light on the multiple roles of COS in protecting against $A \beta$-associated pathology. Given the availability, low toxicity and high bio-tolerance, the use of COS as potential therapeutic agents for the treatment of AD deserves further investigation.

\section{Materials and Methods}

\subsection{Materials}

Cell culture reagents were obtained from Life Technologies (Gaithersburg, MD, USA). Amyloid $\beta$-protein fragments 1-42 (A $\beta 42, \geq 95 \%)$, 3-[4,5-dimethylthiazol-2-yl]-2,5-diphenyltetrazolium bromide (MTT), thioflavin T (ThT), 1,1,1,3,3,3-hexafluoro-2-propanol (HFIP), dimethyl sulfoxide (DMSO) were purchased from Sigma-Aldrich (St. Louis, MO, USA). COS (average molecular weight $<$ 1000, DD. 91.3\%) were obtained from Dalian Glycobio Co., Ltd. (Dalian, China). Annexin V: FITC Apoptosis Detection Kit I was obtained from BD Biosciences (San Jose, CA, USA). All other reagents were of the highest grade available and purchased from Sigma-Aldrich unless otherwise indicated.

\subsection{Peptide Preparation}

$\mathrm{A} \beta 42$ was obtained in lyophilized form on arrival. To disrupt the preformed $A \beta$ aggregates into monomers, $A \beta 42$ was initially dissolved in HFIP to a final concentration of $1 \mathrm{mM}$, sonicated for $30 \mathrm{~min}$, 
and then centrifuged at $14,000 \mathrm{rpm}$ for $20 \mathrm{~min}$ at $4{ }^{\circ} \mathrm{C}$ to remove any pre-existing aggregates [27]. The HFIP was allowed to evaporate in the fume hood overnight, while any remaining trace of HFIP was spin-vacuumed using a Thermo Savant Speed-Vac system (Waltham, MA, USA). The peptide film was stored at $-80{ }^{\circ} \mathrm{C}$ until use.

\subsection{Inhibition and Disruption Assay}

HFIP-treated samples (homogeneous solution of $A \beta$ monomers) were completely resuspended to $5 \mathrm{mM}$ in DMSO by pipette mixing followed by bath sonication for $5 \mathrm{~min}$. A $\beta$ fibrils were prepared by diluting $\mathrm{A} \beta$ in DMSO to $100 \mu \mathrm{M}$ in $10 \mathrm{mM} \mathrm{HCl}$, vortexing for $30 \mathrm{~s}$, and then centrifuged at 14,000 rpm for $30 \mathrm{~min}$ at $4{ }^{\circ} \mathrm{C}$ to remove any existing oligomers. The supernatant was collected for further experiments [14]. For inhibition experiments, COS stock solution was dissolved in freshly prepared A $\beta 42$ monomer solutions to a final concentration of 2.5 and $5.0 \mathrm{mg} / \mathrm{mL}$ (with $\mathrm{A} \beta$ final concentration of $50 \mu \mathrm{M}$ ), or further diluted to a required concentration. For disruption assay, A $\beta$ fibrils were prepared by incubating at $37^{\circ} \mathrm{C}$ for $48 \mathrm{~h}$, which is long enough to form mature fibrils. To examine the effect of $\operatorname{COS}$ on $\mathrm{A} \beta$ fibrils disruption, $\operatorname{COS}$ were coincubated with $\mathrm{A} \beta$ fibrils at $37^{\circ} \mathrm{C}$ for another $48 \mathrm{~h}$.

\subsection{Circular Dichroism Spectroscopy (CD)}

Secondary structural changes in peptide were detected using CD spectroscopy [28]. All measurements were performed in quartz cuvette cells with a path length of $1 \mathrm{~mm}$ and scanned with a J-810 CD spectropolarimeter (Jasco, Tokyo, Japan). The stock peptide was spin-vacuumed then dissolved in $10 \mathrm{mM}$ phosphate buffer ( $\mathrm{pH}$ 7.4), and the final concentration of $\mathrm{A} \beta$ in each sample was $50 \mu \mathrm{M}$. CD measurements were carried out between 190 and $240 \mathrm{~nm}$ using the following parameters: 2-nm bandwidth, $20 \mathrm{~nm} / \mathrm{min}$ run speed, 0.5-nm step size, and 2-s response time. Direct CD measurements $\left(\theta\right.$, in mdeg) were converted to molar ellipticity, $(\theta)\left(\mathrm{deg} \cdot \mathrm{cm}^{2} \cdot \mathrm{mol}^{-1}\right)$ using the formula $(\theta)=\theta / 10 \times c \times l$, where $c$ represent the molar concentration and $l$ the path length $(\mathrm{cm})$. Background values for each test were subtracted from the corresponding values of each sample, and the spectra were smoothed using the Jasco software FFT filter function and converted to molar ellipticity.

\subsection{Transmission Electron Microscopy (TEM)}

The morphological changes of $A \beta$ aggregation in the presence or absence of COS were characterized by TEM. Samples used in the CD spectroscopy were diluted in 1:5 ratios and taken for TEM measurements at different time points to correlate A $\beta 42$ morphological changes with A $\beta$ growth kinetics. Each sample was spotted onto glow-discharged, Formvar-carbon coated 300 mesh copper grids (Ted Pella Inc., Redding, CA, USA) for $1 \mathrm{~min}$, dried, and then negatively stained with 2\% uranyl acetate. The excess liquid was blotted, and the grid was allowed to dry. The prepared samples were then examined with a JEM-1230 electron microscope (JEOL, Tokyo, Japan) at the voltage of $80 \mathrm{kV}$.

\subsection{Thioflavine T (ThT) Fluorescence Assay}

A $\beta 42$ fibrillization and preformed fibrils disruption in the presence or absence of COS was monitored using ThT fluorescence assay. ThT solution was diluted in Tris-buffer $(\mathrm{pH}=7.4)$ to a final 
concentration of $10 \mu \mathrm{M}$. The fluorescence intensity was measured at $37{ }^{\circ} \mathrm{C}$ using a Varioskan multimode microplate spectrophotometer (Thermo, Waltham, MA, USA) under kinetic fluorometric mode. Measurements were carried out at an excitation wavelength of $450 \mathrm{~nm}$ and an emission of $485 \mathrm{~nm}$ [28]. To account for background fluorescence, the fluorescence intensity from solution without A $\beta 42$ was subtracted from solution containing $A \beta 42$.

\subsection{Primary Neuronal Culture and Treatment}

This study was performed in compliance with the Public Health Service Policy on Humane Care and Use of Laboratory Animals. Cortical neuronal cultures were carried out as described previously [15]. Briefly, embryonic day 17 Sprague Dawley rat cortices were dissociated and suspended in fresh neurobasal medium plus 2\% B27 supplements, then plated onto poly-D-lysine-coated 96-well culture plates at a density of $5 \times 10^{4}$ cells per well and maintained at $37{ }^{\circ} \mathrm{C}$ in $\mathrm{CO}_{2}$ incubator. This method resulted in cultures highly enriched for neurons ( $>95 \%$ purity) as assessed by immunostaining against MAP-2. Neurons were allowed to mature for 7 days before commencing treatment. For treatment, A $\beta 42$ samples with or without $\operatorname{COS}$ were diluted to a concentration of $5 \mu \mathrm{M}$ in neurobasal medium, and finally added to the cultures for $48 \mathrm{~h}$.

\subsection{Cell Viability Assay}

The cellular viability was measured by MTT reduction. Briefly, MTT solution in phosphate-buffered saline (PBS) was added to a final concentration of $0.5 \mathrm{mg} / \mathrm{mL}$. The plate was incubated at $37{ }^{\circ} \mathrm{C}$ for additional $4 \mathrm{~h}$. Finally, the medium containing MTT was removed and $100 \mu \mathrm{L}$ DMSO was added to each well, and agitated at room temperature for $30 \mathrm{~min}$ to dissolve crystals. The amount of MTT formazan was determined by measuring the absorbance at $570 \mathrm{~nm}$, with $630 \mathrm{~nm}$ as a reference.

\subsection{Flow Cytometric Detection of Cell Apoptosis}

Cell apoptosis was detected by flow cytometry using Annexin V Apoptosis Detection Kit according to the manufacturer's protocol. Briefly, cultured neurons were washed twice by PBS, trypsinized and centrifuged at $1000 \mathrm{rpm}$ for $5 \mathrm{~min}$, and the pellet was resuspended in binding buffer with FITC-conjugated Annexin V and PI for $15 \mathrm{~min}$ at room temperature, and then analyzed by flow cytometry (FACSCalibur; BD Biosciences, San Jose, CA, USA).

\subsection{Statistical Analysis}

The results were expressed as means \pm SEM of at least three independent experiments. Statistical evaluation was performed by one-way analysis of variance (ANOVA) followed by Student-Newman-Keuls as post-hoc test. $p<0.05$ was considered to be statistically significant.

\section{Acknowledgments}

This work was supported by grants from NSFC Project 31071512, and grants from Scientific Research Common Program of Beijing Municipal Commission of Education (SQKM201511417013). 
We gratefully thank Lin Yang (Institute of Genetics and Developmental Biology, Chinese Academy of Sciences) for her technical help with the use of TEM.

\section{Author Contributions}

Xueling Dai, Wanqi Hou, Yaxuan Sun and Zhaolan Gao performed the experiments and analyzed the data; Shigong Zhu and Zhaofeng Jiang designed the experiments; Xueling Dai and Zhaofeng Jiang wrote the manuscript. All authors read and approved the final manuscript.

\section{Conflicts of Interest}

The authors declare no conflict of interest.

\section{References}

1. Medeiros, R.; Baglietto-Vargas, D.; LaFerla, F.M. The role of tau in Alzheimer's disease and related disorders. CNS Neurosci. Ther. 2011, 17, 514-524.

2. Ballard, C.; Gauthier, S.; Corbett, A.; Brayne, C.; Aarsland, D.; Jones, E. Alzheimer's disease. Lancet 2011, 377, 1019-1031.

3. Selkoe, D.J. Preventing Alzheimer's disease. Science 2012, 337, 1488-1492.

4. Walker, L.C.; Jucker, M. Amyloid by default. Nat. Neurosci. 2011, 14, 669-670.

5. Seltzer, B. Cholinesterase inhibitors in the clinical management of Alzheimer's disease: Importance of early and persistent treatment. J. Int. Med. Res. 2006, 34, 339-347.

6. Van Marum, R.J. Current and future therapy in Alzheimer's disease. Fundam. Clin. Pharmacol. 2008, 22, 265-274.

7. Williams, P.; Sorribas, A.; Howes, M.J. Natural products as a source of Alzheimer's drug leads. Nat. Prod. Rep. 2011, 28, 48-77.

8. Jana, S.; Sen, K.K.; Basu, S.K. Chitosan derivatives and their application in pharmaceutical fields. Int. J. Pharm. Res. 2011, 3, 1-8.

9. Liu, H.T.; Li, W.M.; Huang, P.; Chen, W.J.; Liu, Q.S.; Bai, X.F.; Yu, C.; Du, Y.G. Chitosan oligosaccharides inhibit TNF- $\alpha$-induced VCAM-1 and ICAM-1 expression in human umbilical vein endothelial cells by blocking p38 and ERK1/2 signaling pathways. Carbohydr. Polym. 2010, 81, 49-56.

10. Fang, I.M.; Yang, C.H.; Yang, C.M.; Chen, M.S. Chitosan oligosaccharides attenuates oxidative-stress related retinal degeneration in rats. PLOS ONE 2013, 8, e77323.

11. Pangestuti, R.; Kim, S.-K. Marine-derived bioactive materials for neuroprotection. Food Sci. Biotechnol. 2013, 22, 1-12.

12. Azuma, K.; Osaki, T.; Minami, S.; Okamoto, Y. Anticancer and anti-inflammatory properties of chitin and chitosan oligosaccharides. J. Funct. Biomater. 2015, 6, 33-49.

13. Dai, X.; Chang, P.; Zhu, Q.; Liu, W.; Sun, Y.; Zhu, S.; Jiang, Z. Chitosan oligosaccharides protect rat primary hippocampal neurons from oligomeric $\beta$-amyloid 1-42-induced neurotoxicity. Neurosci. Lett. 2013, 554, 64-69. 
14. Petrlova, J.; Kalai, T.S.; Maezawa, I.; Altman, R.; Harishchandra, G.; Hong, H.-S.; Bricarello, D.A.; Parikh, A.N.; Lorigan, G.A.; Jin, L.-W.; et al. The influence of spin-labeled fluorene compounds on the assembly and toxicity of the A $\beta$ peptide. PLOS ONE 2012, 7, e35443.

15. Jiang, T.; Zhi, X.L.; Zhang, Y.H.; Pan, L.F.; Zhou, P. Inhibitory effect of curcumin on the Al(III)-induced A $\beta 42$ aggregation and neurotoxicity in vitro. Biochim. Biophys. Acta 2012, 1822, 1207-1215.

16. Jan, A.; Hartley, D.M.; Lashuel, H.A. Preparation and characterization of toxic A $\beta$ aggregates for structural and functional studies in Alzheimer's disease research. Nat. Protoc. 2010, 5, 1186-1209.

17. Seeman, P.; Seeman, N. Alzheimer's disease: $\beta$-Amyloid plaque formation in human brain. Synapse 2012, 65, 1289-1297.

18. Anand, R.; Gill, K.D.; Mahdi, A.A. Therapeutics of Alzheimer's disease: Past, present and future. Neuropharmacology 2014, 76, 27-50.

19. Wang, Q.; Yu, X.; Patel, K.; Hu, R.; Chuang, S.S.; Zhang, G.; Zheng, J. Tanshinones inhibit amyloid aggregation by amyloid- $\beta$ peptide, disaggregate amyloid fibrils, and protect cultured cells. ACS Chem. Neurosci. 2013, 19, 1004-1015.

20. Minicozzi, V.; Chiaraluce, R.; Consalvi, V.; Giordano, C.; Narcisi, C.; Punzi, P.; Rossi, G.C.; Morante, S. Computational and experimental studies on $\beta$-sheet breakers targeting A $\beta 1-40$ fibrils. J. Biol. Chem. 2014, 289, 11242-11252.

21. Ruggeri, F.S.; Adamcik, J.; Jeong, J.S.; Lashuel, H.A.; Mezzenga, R.; Dietler, G. Influence of the $\beta$-sheet content on the mechanical properties of aggregates during amyloid fibrillization. Angew. Chem. Int. Ed. Engl. 2015, 54, 2462-2466.

22. Liu, R.; Barkhordarian, H.; Emadi, S.; Park, C.B.; Sierks, M.R. Trehalose differentially inhibits aggregation and neurotoxicity of $\beta$-amyloid 40 and 42. Neurobiol. Dis. 2005, 20, 74-81.

23. Liu, F.-F.; Ji, L.; Dong, X.-Y.; Sun, Y. Molecular insight into the inhibition effect of trehalose on the nucleation and elongation of amyloid $\beta$-peptide oligomers. J. Phys. Chem. B 2009, 113, 11320-11329.

24. Benilova, I.; Karran, E.; de Strooper, B. The toxic A $\beta$ oligomer and Alzheimer's disease: An emperor in need of clothes. Nat. Neurosci. 2012, 15, 349-357.

25. Yin, F.; Liu, J.; Ji, X.; Wang, Y.; Zidichouski, J.; Zhang, J. Silibinin: A novel inhibitor of A $\beta$ aggregation. Neurochem. Int. 2011, 58, 399-403.

26. Wang, S.W.; Wang, Y.J.; Su, Y.J.; Zhou, W.W.; Yang, S.G.; Zhang, R.; Zhao, M.; Li, Y.N.; Zhang, Z.P.; Zhan, D.W.; et al. Rutin inhibits $\beta$-amyloid aggregation and cytotoxicity, attenuates oxidative stress, and decreases the production of nitric oxide and proinflammatory cytokines. Neurotoxicology 2012, 33, 482-490.

27. Stine, W.B.; Dahlgren, K.N.; Krafft, G.A.; LaDu, M.J. In vitro characterization of conditions for amyloid- $\beta$ peptide oligomerization and fibrillogenesis. J. Biol. Chem. 2003, 278, 11612-11622.

28. Dai, X.; Chang, P.; Liu, W.; Xu, K.; Sun, Y.; Zhu, S.; Jiang, Z. A $\beta-40$ Y10F increases $\beta$ fibrils formation but attenuates the neurotoxicity of amyloid- $\beta$ peptide. Int. J. Mol. Sci. 2012, 13, 5324-5337.

(C) 2015 by the authors; licensee MDPI, Basel, Switzerland. This article is an open access article distributed under the terms and conditions of the Creative Commons Attribution license (http://creativecommons.org/licenses/by/4.0/). 